\title{
La política universitaria del gobierno Bolsonaro y las reacciones de la comunidad científica brasileña
}

Hoy en día estamos viviendo uno de los momentos más turbulentos de la historia republicana brasileña y, particularmente en lo relativo a las relaciones entre el gobierno nacional y las universidades públicas. Desde su nombramiento, en abril de 2019, el Ministro de la Educación Abraham Weintraub viene haciendo innumerables manifestaciones en contra de la comunidad académica brasileña. En una de sus declaraciones más recientes, a finales de noviembre, incluso denunció en la prensa la diseminación de plantaciones de marihuana en los campus de las universidades brasileñas, aunque sin presentar pruebas.

Otro ejemplo. A fines de abril de 2019, el Ministro declaró que recortaría fondos de las universidades que estaban promoviendo alboroto en sus campus; antes de eso el gobierno nacional había intentado impedir que se impartiera un curso de grado en la Universidad de Brasilia sobre el golpe de Estado que derrumbó a la Presidente Dilma Rousseff en 2016. Identificó a la mencionada Universidad de Brasilia, junto con la Universidad Federal Fluminense y la Universidad Federal de Bahía como las instituciones en las cuales serepetían desórdenes; luego, declaró que las mismas tendrían reducción de recursos federales.

Días más tarde, ante innumerables críticas por parte de la opinión pública, el Ministro informó un cambio de idea: los recortes serían ahora para todas las universidades brasileñas, impactando en casi treinta por ciento del presupuesto de gran parte de las instituciones del país.

Como consecuencia, desde agosto de 2019 ha habido una gran movilización de la comunidad académica en todo el país, que no sólo critica la reducción de recursos, sino pone en tela de juicio el intento del gobierno nacional de censurar la actividad científica, intentando interferir no sólo en la gestión de las universidades, sino en los contenidos didácticos presentados por los profesores en sus clases. En el primer semestre de 2019 hubo varias declaraciones del Ministro Weintraub sugiriendo que los alumnos filmasen las clases en las universidades y denunciasen a docentes que impartiesen contenidos considerados impropios por estudiantes o sus familias.

La universidad brasileña jugó un papel esencial en los momentos más importantes de la nación. Ya sea creando un apoyo científico tecnológico enfocado en áreas estratégicas, o movilizando a la comunidad universitaria en manifestaciones a favor o en contra de determinadas políticas públicas, la universidad fue un actor clave en la afirmación de un modelo de desarrollo nacional capaz de potencializar la riqueza del país (Cunha, 2007).

Por cierto, algo semejante ha pasado en parte importante de América Latina, donde la universidad fue responsable por “(...) diseñar proyectos nacionales de desarrollo o modernización, liderados por el Estado, que tenían como objetivo crear o profundizar la

\footnotetext{
* Doctor en Ciencias Políticas y Sociología. Profesor Departamento de Ciencia Política, Universidade Federal do Rio Grande do Sul (UFRGS, Brasil). Correo electrónico: alfredogugliano@hotmail.com
} 
coherencia y la cohesión del país como un espacio económico, social y cultural, territorio geográficamente bien definido en su conjunto" (Santos, 2008:45).

En Brasil, desde las primeras décadas del siglo XIX, la Universidad fue central en la formación de la élite responsable de la dirección de las instituciones políticas del país. Una dimensión que sólo se expandió en el transcurso del siglo XX, en particular con la participación de la comunidad universitaria en la redemocratización de 1945 y la movilización por la autonomía energética del país, con el slogan "o petróleo é nosso". Algunos años más tarde la universidad volvió a cumplir destacada función tanto en la resistencia a la dictadura militar de 1964, como en la lucha por la redemocratización, muy especialmente en las jornadas por elecciones directas para la Presidencia de la nación en 1984.

Igualmente fue activa en las caminatas que legitimaron el impeachment del Presidente Fernando Collor de Mello, a finales de 1992, así como en las jornadas del 2013 que fueron la base del desarrollo de un movimiento nacional contra la corrupción política en Brasil, fenómeno fundamental para comprender lo que pasó en el país en la segunda década del siglo XXI.

El compromiso político de las universidades públicas brasileñas jamás estuvo vinculado a partidos políticos (Fiori, 1986). Siempre fue considerado como una expresión natural de una institución que, en los diferentes campos científicos, estuvo dirigida hacia la producción de un conocimiento destinado a mejorar la sociedad, en hacer del mundo un lugar mejor, donde las personas puedan vivir con dignidad. Esa es la idea fuerza que mueve la rutina de millares de científicos, tanto dentro como fuera del país, en el trabajo diario en clases, laboratorios y centros de investigación.

Desde sus comienzos la universidad siempre ha entendido a la política en sentido amplio. Aunque existan miembros del cuerpo académico que pueden estar vinculados a proyectos políticos partidistas, el concepto de política frecuentemente fue interpretado dentro de las universidades como un bien público, concebido como el arte del relacionamiento cívico, republicano. Según la perspectiva de Hannah Arendt (1998) la política entendida como algo esencial a la vida humana, el componente que vincula el individuo a la sociedad.

Así es como gran parte de la sociedad ve a las instituciones académicas: un espacio para desarrollar políticas públicas independientes de los gobiernos y sus ideologías, comprometidas con el futuro de la nación.

Incluso con todas las diferencias existentes entre los partidos políticos brasileños, la autonomía de las universidades siempre fue respetada por todos los Presidentes que gobernaron el país desde la redemocratización hasta 2018, cuando un nuevo gobierno identificado con políticas conservadoras gana las elecciones y aumenta la propensión gubernamental por intervenir de manera más fuerte en la gestión de las universidades públicas brasileñas. Con el Presidente Jair Bolsonaro las relaciones entre las universidades y el gobierno sufrieron un cambio importante, impactando en la preservación de un posicionamiento autónomo e independiente por parte de las universidades, especialmente de sus profesores y alumnos.

La entrevista del Ministro de Educación, Abraham Weintraub, en mayo de 2019, justificando recortes en el presupuesto universitario, ilustra esta nueva postura adoptada por el gobierno de la nación. Dice el Ministro: "Las universidades que, en lugar de tratar de mejorar el rendimiento académico, están haciendo quilombo, tendrán reducidos sus fondos. Deben hacer la tarea para casa: publicación científica, evaluaciones positivas, buena posición en los rankings" (O Estado de São Paulo, 30 de abril de 2019). Una declaración demasiado hostil especialmente si consideramos que las principales universidades brasileñas generalmente ocupan posiciones importantes en las evaluaciones internacionales de la enseñanza superior.

En las semanas posteriores a esta declaración, se hicieron recortes en los presupuestos universitarios; el presupuesto en ciencia y tecnología tuvo una disminución del más de $40 \%$ en 2019, fueron canceladas más de 8000 becas de postgrado. Con todo, no fue presentado ningún estudio que justificase actitud tan radical, ni se demostraron gastos irregulares en las 
universidades, ni fallas relacionadas con la producción científica y tecnológica de las instituciones.

Las consecuencias de la reducción de los presupuestos universitarios impactaron en un amplio número de cuestiones, desde el pago de elementos básicos para el funcionamiento de las instituciones, como cuentas de agua y luz, hasta la compra de equipos para los laboratorios, la presentación de papers en congresos académicos, la investigación de campo, etc.

Igualmente fue vulnerada la democracia en las universidades. Varios rectores elegidos por la comunidad académica no fueron nombrados por el gobierno, sino que fueron juramentados candidatos que, aunque no obtuvieron la mayoría de los votos, eran de la confianza del equipo ministerial.

Concomitantemente con la reducción abrupta de los recursos para las universidades, el gobierno presentó en los últimos meses de 2019 un nuevo proyecto destinado a reformar las instituciones de enseñanza superior en el país. La propuesta llamada en portugués "future-se", algo así como prepárense para el futuro, entre otras proposiciones estimula la vinculación de las universidades públicas con empresas de capital privado y la creación de consejos externos de administración, formados por representantes de la sociedad posiblemente designados por el gobierno, para dirigir a las universidades brasileñas.

Desde septiembre varios consejos universitarios vinculados a diferentes universidades públicas se han opuesto a esta propuesta. Los principales argumentos que respaldan esta posición son que el "future-se" quiebra con la autonomía universitaria al aceptar la posibilidad de una gestión externa en las instituciones. También fomenta la reducción de las inversiones del Estado en educación, vinculando la financiación universitaria de las instituciones públicas a las contribuciones de las organizaciones económicas centradas en el beneficio privado. En suma, una propuesta que contribuye a desestructurar el sistema público de educación, beneficiando a las universidades privadas y reduciendo los espacios democráticos en el interior de la gestión universitaria.

Como resultado de estas proposiciones la universidad brasileña vive un fuerte proceso de movilización, que está en gran medida vinculado a la defensa de la ciencia y tecnología brasileña, la garantía de autonomía de la nación frente a un marco económico internacional en el cual el desarrollo tecnológico está directamente relacionado con la importancia de cada país en el escenario político internacional.

En la actualidad, la movilización política de la comunidad universitaria se ha destacado en dos aspectos. En primer lugar, debido al creciente debate dentro de las universidades públicas sobre las políticas educativas del Gobierno Bolsonaro y sus implicaciones.

En este universo se están discutiendo aspectos relacionados con la libertad de cátedra y la necesidad de que las clases puedan ser desarrolladas sin ningún tipo de censura o coacción sobre la actividad docente. Por ejemplo, la posibilidad de continuar manteniendo asignaturas e investigaciones sobre un conjunto de contenidos usualmente discutidos en el campo de las humanidades, como cuestiones de género y derechos humanos, pero que sufren fuerte resistencia por parte de los dirigentes gubernamentales. Además, el resguardo de la democracia en las universidades está siendo visto como elemento esencial de la propia actividad científica en el país. Garantizar que la comunidad de profesores, estudiantes y de otros trabajadores vinculados a la universidad, puedan participar de los debates de las políticas internas de las instituciones, así como elegir los dirigentes de las facultades y universidades, es una conquista que debe ser mantenida.

En segundo lugar, la movilización universitaria llegó a las calles de las principales ciudades brasileñas entre septiembre y octubre, convirtiéndose en un canal desde el cual toda la sociedad expresó su preocupación sobre la situación de la educación pública en el país, mostrando insatisfacción delante de proyectos presentados por el gobierno de la nación que apuntan no sólo a una posible privatización de las universidades, sino que promueven una clara 
ideologización de los contenidos presentados en clases, rescatando una doctrina cívico-militar identificada con el período de la dictadura militar en el país.

Además de jornadas de protesta contra las políticas gubernamentales, estas marchas representaron el deseo de la comunidad académica y de parte de la sociedad brasileña de garantizar una educación pública de calidad, con universidades fuertes y capaces de desarrollar actividades en el campo de la ciencia y tecnología de acuerdo con las libertades previstas en el Estado democrático de derecho.

Como resultado de las movilizaciones a finales de octubre el Ministerio de la Educación liberó gran parte de los recursos financieros que habían sido suspendidos a comienzos del año, siendo que obligatoriamente deberán ser utilizados hasta finales del 2019. Esa liberación aumenta más la sensación de que los recortes presupuestarios no fueron establecidos por problemas financieros, pero sí por desacuerdos políticos del gobierno con las universidades públicas.

Todavía es prematuro predecir una solución para esta situación. Sin embargo, la ofensiva del gobierno sobre algunos de los logros históricos de la universidad brasileña tuvo como principal consecuencia el despertar de la comunidad académica a la gravedad del momento político que vivimos en el país. Y, sin duda, algo nuevo está en el aire. En las multitudinarias caminatas en defensa de la educación reuniendo a docentes, estudiantes, trabajadores, artistas, amas de casa, indígenas, es decir, ciudadanos con las más diversas características, existe la convicción de que la autonomía universitaria y la democracia son elementos esenciales al desarrollo del país.

\section{Referencias}

ARENT, H. (2006). O que é política?, Bertrand, Rio de Janeiro

CUNHA, L. A. (2007). A universidade crítica: o ensino superior na república populista, Editora UNESP, São Paulo

FIORI, E. M. (1986). Conscientização e educação, Educação e Realidade, Porto Alegre, 11 (1), pp.. 3-10 jan./jun

SANTOS, B. S. (2008). A Universidade no século XXI: para uma universidade nova, Almedina, Coimbra 